\title{
Acupuncture to Improve Symptoms for Stable Angina: Protocol for a Randomized Controlled Trial
}

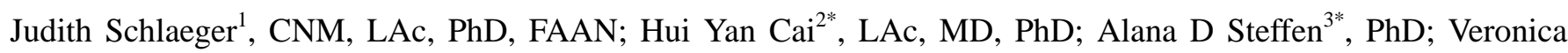
Angulo $^{1^{*}}$, BA; Adhir R Shroff ${ }^{4 *}, \mathrm{MD}, \mathrm{MPH}$; Joan E Briller ${ }^{4 *}$, MD; Debra Hoppensteadt ${ }^{5 *}, \mathrm{PhD}$; Glorieuse Uwizeye ${ }^{6}$, RN, MScN; Heather A Pauls ${ }^{7 *}$, MPH; Miho Takayama ${ }^{8^{*}}, \mathrm{RN}, \mathrm{LAc}, \mathrm{PhD}$; Hiroyoshi Yajima ${ }^{8^{*}}, \mathrm{LAc}, \mathrm{PhD}$; Nobuari Takakura $^{8 *}$, LAc, PhD; Holli A DeVon ${ }^{6}$, RN, PhD, FAHA, FAAN

${ }^{1}$ Department of Women, Children and Family Health Science, College of Nursing, University of Illinois at Chicago, Chicago, IL, United States

${ }^{2}$ Department of Acupuncture and Oriental Medicine, National University of Health Sciences, Lagrange, IL, United States

${ }^{3}$ Department of Health Systems Science, College of Nursing, University of Illinois at Chicago, Chicago, IL, United States

${ }^{4}$ Department of Cardiology, College of Medicine, University of Illinois at Chicago, Chicago, IL, United States

${ }^{5}$ Department of Pathology and Pharmacology, Loyola University Medical Center, Maywood, IL, United States

${ }^{6}$ Department of Biobehavioral Health Science, College of Nursing, University of Illinois at Chicago, Chicago, IL, United States

${ }^{7}$ Office of Research Facilitation, College of Nursing, University of Illinois at Chicago, Chicago, IL, United States

${ }^{8}$ Department of Acupuncture and Moxibustion, Tokyo Ariake University of Medical and Health Sciences, Tokyo, Japan

* these authors contributed equally

\section{Corresponding Author:}

Judith Schlaeger, CNM, LAc, PhD, FAAN

Department of Women, Children and Family Health Science

College of Nursing

University of Illinois at Chicago

845 S Damen Avenue

Suite 812 , MC 802

Chicago, IL, 60612

United States

Phone: 13124134669

Fax: 13129968871

Email: jschlaeg@uic.edu

\section{Abstract}

Background: Acupuncture has demonstrated physiologic analgesic effects in Chinese patients with stable angina. One proposed mechanism of action for these analgesic effects is the downregulation of M1 macrophages, interleukin 1 beta, interleukin-6, interleukin-18, and tumor necrosis factor alpha.

Objective: This study aims to test a 10-session, 5-week acupuncture treatment protocol as a complementary therapy for symptoms of stable angina for American patients, who vary from Chinese patients in health care systems and other salient variables.

Methods: We are conducting a randomized controlled trial (RCT) of 69 adults ( 35 assigned to initial acupuncture and 34 to an attention control condition) with a medically confirmed diagnosis of stable angina, whose pain and associated symptoms have not been controlled to their satisfaction with guideline-directed medical management. Participants in the experimental group will receive a standardized traditional Chinese medicine point prescription. The attention control group will view non-pain-related health education videos over 5 weeks equal to the 10 hours of treatment for the acupuncture group. Participants will complete the McGill Pain Questionnaire and the Seattle Angina Questionnaire-7, as well as have inflammatory cytokines measured at baseline and study completion. The primary outcomes are anginal pain and quality of life.

Results: This study has been funded over 2 years by the National Institutes of Health, National Institute for Nursing Research. We are currently recruiting and expect to have initial results by December 2020.

Conclusions: We will generate data on feasibility, acceptability, effect sizes, and protocol revisions for a future fully powered RCT of the protocol. Findings will help determine if patients with persistent ischemic symptoms experience a proinflammatory state and hyperalgesia caused by multiple neural and immune processes not always relieved with medication. 
International Registered Report Identifier (IRRID): DERR1-10.2196/14705

(JMIR Res Protoc 2019;8(7):e14705) doi: 10.2196/14705

\section{KEYWORDS}

acupuncture; stable angina; ischemic heart disease; complementary medicine

\section{Introduction}

\section{Background}

The significance of managing the symptoms of stable angina is critical, given that stable angina increases the risk of acute coronary syndrome for women and mortality for men [1,2]. Camm et al [3] found that one-third of patients with stable angina have suboptimal management of their pain. Guideline-directed therapy for stable angina includes nitrates, beta-blockers, calcium channel blockers, and angiotensin-converting enzyme inhibitors [4]. These drugs can have side effects such as headache, flushing, fatigue, and cough, which can lead to nonadherence and reduced symptom control [5]. Acupuncture, if effective, may help control angina pain for patients nonadherent to medications because of debilitating side effects.

Acupuncture has demonstrated physiologic analgesic effects. It regulates the autonomic nervous system and reduces sympathetic stimulation to the heart and vasculature by modulating the midbrain, releasing endorphins and dynorphins, with a resultant decrease in production of norepinephrine and epinephrine [6-12]. These processes impact the cardiovascular system by reducing blood pressure, heart rate, and arrhythmias [13] centrally mediated in the brainstem [12]. Acupuncture may help relieve pain by activating mu opioid receptors, which decreases pain [6], increasing serum beta endorphins [7] and downregulating M1 macrophages, interleukin (IL) 1 beta, IL-6, ILin-18, and tumor necrosis factor (TNF) alpha [14], which reduces inflammation [15]. The mechanism of action is stimulation of a neuronal circuit that detects inflammatory mediators and releases dopamine, inhibiting release of inflammatory cytokines [15]. No studies have directly examined the physiologic effects of acupuncture on the cardiovascular system for the treatment of angina.

In traditional Chinese medicine (TCM), $q i$ is the vital energy flowing within and surrounding the body [16]. Disorders of $q i$ or blood can result in pain, and $q i$ can be deficient or in excess (stagnant or obstructed). The channels through which $q i$ and blood flow in the body are called meridians [17]. Acupuncture needles are inserted into acupuncture points, which access the meridians and promote the circulation of qi and blood [18]. Deficient $q i$ and/or blood is strengthened (tonified); excess qi and/or blood is moved to reduce stagnation or obstruction $[19,20]$. Thus, the body becomes balanced, and pain and other symptoms are reduced [18,19]. In the TCM model, it is theorized that the pain, shortness of breath, and fatigue of stable angina result when there is an excess or a deficiency in $q i$ and/or blood in the meridians that flow through and around the heart [20-22].

\section{Objectives}

The scientific premise of our study is that patients with persistent ischemic symptoms experience a proinflammatory state and hyperalgesia caused by multiple neural and immune processes not always relieved with medication [15]. The aims of our study are to test the feasibility of a 10-session, 5-week acupuncture treatment protocol as a complementary therapy for symptoms of stable angina in American patients and to estimate effect sizes for change from pre- to postintervention scores for pain/symptoms, inflammatory cytokines, functional status, and health-related quality of life (HRQoL) between the acupuncture and control groups. We hypothesize that participants with stable angina will see a reduction in pain following acupuncture [8].

\section{Methods}

\section{Study Design}

This randomized attention-control feasibility study will be conducted at an academic medical center in the Midwest. This design will facilitate the calculation of effect sizes for withinand between-group differences in pain and symptoms. The design is more appropriate than either placebo or sham acupuncture interventions at this stage of investigation because the protocol has not been tested in an American population of patients with stable angina. A placebo control design is not possible because neither the clinician nor participant can be blinded to the acupuncture treatment without the use of double-blind acupuncture needles. Currently, double-blind needles are being produced by hand, tested in small studies, and are not yet commercially available [23,24]. Sham acupuncture, whereby needles are inserted into nonacupuncture points on the body, is inferior to randomized control trial (RCT) with attention control design because needle punctures anywhere on the skin may be considered an ashi point (ie, an active acupuncture point that may have therapeutic effects) $[25,26]$. Following consent and upon enrollment in the study, participants will be randomized 1:1 to the acupuncture or attention control group using a permuted block approach, stratified by biological sex. This study was approved by the University of Illinois at Chicago Institutional Review Board (UIC IRB).

\section{Sample and Setting}

A total of 69 patients with a confirmed diagnosis of stable angina will be included. Only those patients with Canadian Cardiovascular Society (CCS) class I to III will be included. By definition, CCS Grade IV (angina at rest for $>20 \mathrm{~min}$ ) is unstable angina [27], and these patients will not be recruited. We are recruiting participants from the University of Illinois Hospital in Chicago, Illinois. Participants are being recruited from the hospital, clinic, and through campus flyers (Figure 1). 
Figure 1. CONSORT (Consolidated Standards of Reporting Trials) flow chart.

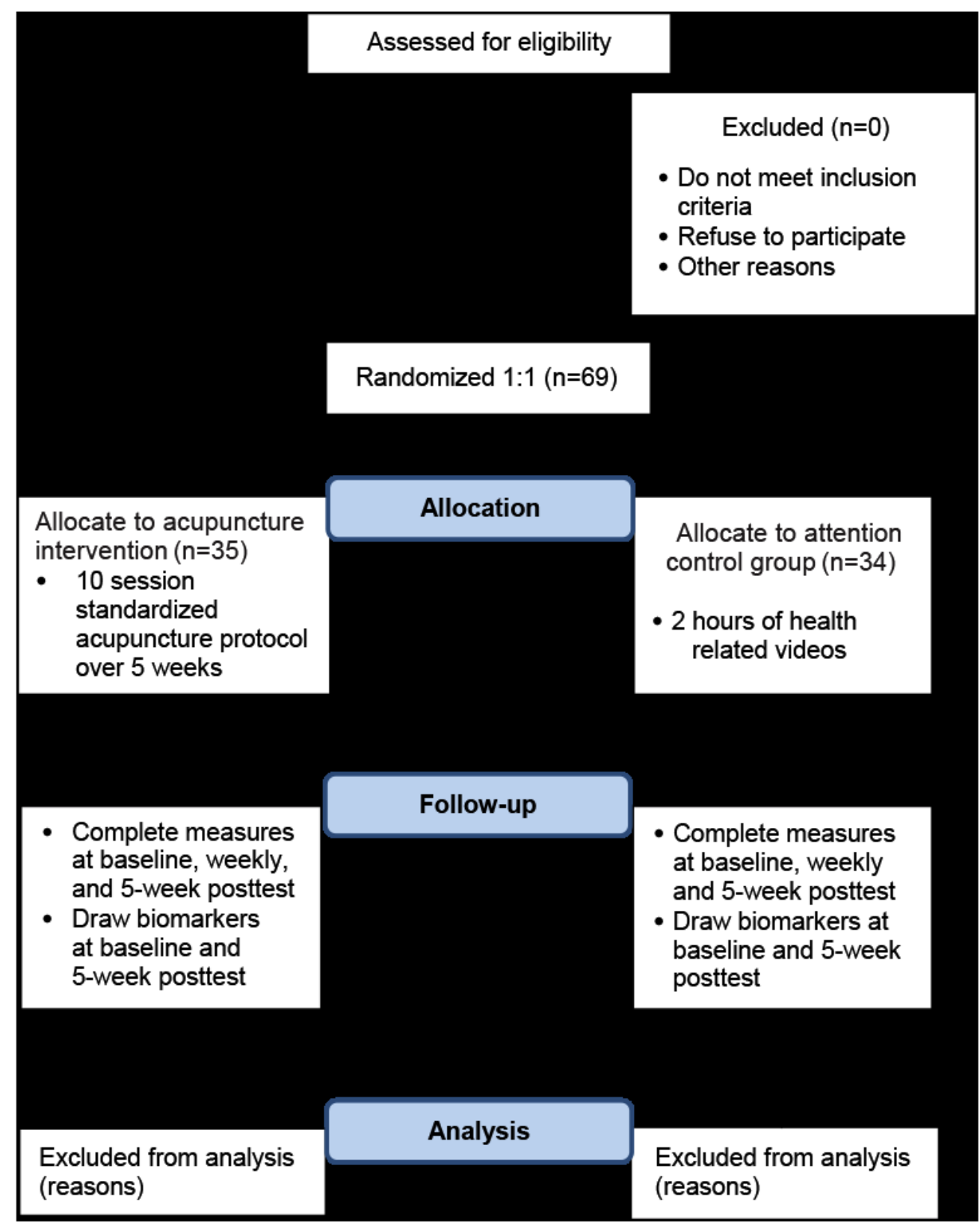

The inclusion and exclusion criteria have been presented in Textboxes 1-3. Participants will continue to take their prescribed medications as directed. The study will be conducted in clinical research exam rooms. Licensed acupuncturists will administer 10 acupuncture treatments to 35 participants in the experimental group (and up to 34 participants in the control group upon study completion). We are using 7 acupuncturists to improve the likelihood that the protocol, and not the acupuncturist, is responsible for the therapeutic effects. All other therapeutic elements will be controlled through our standardized protocol. Acupuncturists will not discuss protocols with the participants.

Textbox 1. Screening tool-inclusion criteria.

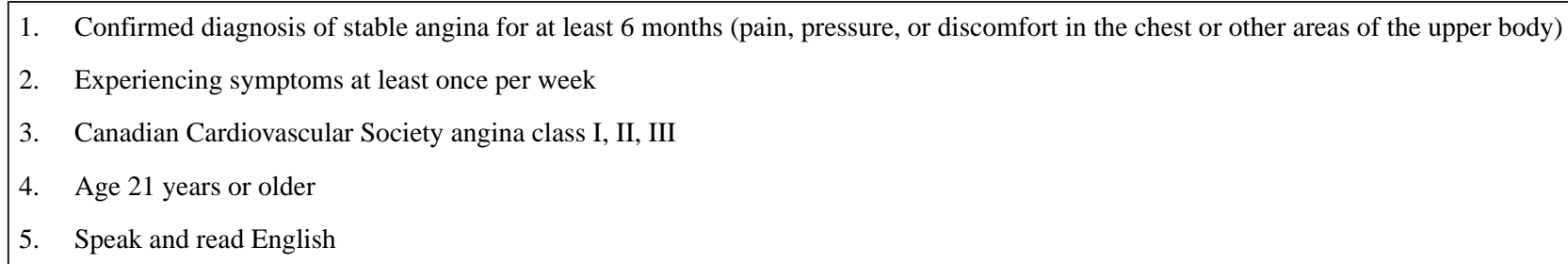


Textbox 2. Screening tool—exclusion criteria.

1. Physical or cognitive limitations that will prevent completion of study tasks (Mini-Cog score 0-2 with abnormal clock face);

2. Exacerbation of heart failure (B-type natriuretic peptide $>500$ or escalating dose of diuretic or admission to hospital for heart failure in past 3 months).

3. Autoimmune dysfunction (use of steroid or prescription anti-inflammatory medications)

4. Moderate to severe chronic obstructive pulmonary disease

- $\quad$ Moderate (treated with short-acting bronchodilators plus antibiotics and/or oral corticosteroids)

- Severe (patient requires hospitalization or visits the emergency room). Severe exacerbations may also be associated with acute respiratory failure

5. Pregnant

6. Use of prescription analgesic medications

7. Currently undergoing treatment with biofeedback, massage, or other acupuncture

Textbox 3. Screening tool—additional questions.
1. Study explained (purpose, rights of participants, informed consent, compensation for time)
2. Has clear understanding of study purpose and requirements
3. Agrees to participate?
4. Reason for declining?
5. If declined: age, sex, race

\section{Measures}

\section{Demographic}

Personal demographic characteristics (age, race/ethnicity, marital status, education, income, employment, and insurance) will be measured.

\section{McGill Pain Questionnaire}

The McGill Pain Questionnaire (MPQ) measures the multidimensionality of pain in 4 domains: sensory (location, intensity, quality, and pattern), affective (anxiety, depression, and fear), evaluative (a comprehensive assessment of the pain), and behavioral (behaviors that worsen or lessen pain) [28]. Current, least, and worst pain in the past 24 hours are scored on a Likert-type scale with $0=$ no pain to $10=$ excruciating pain. The MPQ has been well validated in cardiac populations [29-32].

\section{Upper Body Diagram}

Everts et al's [33] upper body diagram consists of a drawing of the human chest with 9 distinct regions plus the neck, left and right arms, and abdomen. Participants are asked to mark the location of their pain on the diagram. The total pain sites are a location measure and will be used to calculate the multidimensionality of the participant's pain.

\section{Seattle Angina Questionnaire-7}

The Seattle Angina Questionnaire-7 (SAQ-7) consists of 7 items in 4 domains (physical limitation, angina stability, angina frequency, and quality of life) measuring the impact of angina on participants' health status. Item responses are coded sequentially from worst to best status and range from 1 to 6 , except quality of life (range 1-5). Scores are generated for each domain and are scaled 0 to 100 , with 0 denoting the worst and 100, the best possible status [34]. The SAQ-7 has been validated among patients with stable ischemic heart disease, those undergoing coronary interventions, and after acute myocardial infarction [35].

\section{American Heart Association Angina Log}

My Angina Log is a simple 1-page diary used to measure each episode of angina. Participants record the date and times they experienced angina, as well as triggers and treatments [36]. Severity of symptoms are measured on a 1 to 4 scale, with 1 representing mild symptoms and 4 very severe symptoms.

\section{Protocol Acceptability Scale for Treating Angina With Acupuncture}

This satisfaction instrument is a 10 -item instrument used to measure acceptability of the study processes and protocols. Items are measured on a 0 (negative response; ie, did not like acupuncture) to 2 (positive response) scale. The protocol will be deemed to have high acceptability if $80 \%$ of participants score $\geq 16$.

All data except for the American Heart Association (AHA) Angina $\log$ and the chest outline to draw angina pain will be collected via the Research and Electronic Data Capture (REDCap) application, which is a Web-based application for securely building and managing surveys and databases.

\section{Biomarkers of Inflammation}

Proinflammatory cytokines IL-1 beta, IL-2, IL-8, and IL-18; C-reactive protein (CRP) [37,38]; and TNF alpha, the anti-inflammatory cytokines IL-4 and IL-10 [37], and the dual 
pro/anti-inflammatory IL-6 [39] will be measured to determine if there is a significant change from baseline to post study (5 week). Venous blood $(4.5 \mathrm{~mL})$ will be collected in a blue-top collection tube containing $3.2 \%$ sodium citrate. Samples will be processed in our clinical lab. Samples will be centrifuged at room temperature for $15 \mathrm{~min}$; plasma is then removed into 2 cryovials in $1 \mathrm{~mL}$ aliquots and stored in a $-80^{\circ} \mathrm{C}$ freezer immediately. A label with study identification number, date, and time of blood draw will be placed on the cryovials before freezing. Samples will be batch analyzed using high-sensitivity multiplex technologies [40]. Table 1 lists all variables, measures, and data collection time points.

Table 1. Data collection variables, measures, and time points (both groups).

\begin{tabular}{|c|c|c|c|c|}
\hline \multirow[t]{2}{*}{ Variable } & \multirow[t]{2}{*}{ Measure/analyses } & \multicolumn{3}{|c|}{ Data collection points } \\
\hline & & Baseline & $\mathrm{x} 10$ & End \\
\hline \multicolumn{5}{|l|}{ Primary aim 1} \\
\hline Recruitment & $\begin{array}{l}\text { Number of enrolled/number invited to participate; Mini-Cog instrument } \\
\text { (screening) }\end{array}$ & $\mathfrak{J}^{\mathrm{a}}$ & $-\mathrm{b}$ & - \\
\hline Retention & Number retained at study completion/number recruited & $\checkmark$ & - & $\checkmark$ \\
\hline Completion & Number of time points completed (11 total) & - & - & $\checkmark$ \\
\hline Acceptability & Protocol Acceptability Scale for Treating Angina with Acupuncture & - & - & $\checkmark$ \\
\hline \multicolumn{5}{|l|}{ Primary aim 2} \\
\hline Demographic data & Demographic data form & $\checkmark$ & - & - \\
\hline Effect size & $\begin{array}{l}\text { Intraindividual effect: mean prescores minus mean postscores/pooled SD. } \\
\text { Group effect: mean differences in scores between acupuncture and attention } \\
\text { control group }\end{array}$ & $\checkmark$ & - & $\checkmark$ \\
\hline \multicolumn{5}{|l|}{ Exploratory aim } \\
\hline Biomarkers & $\begin{array}{l}\text { Interleukin } 1 \text { beta, } 2,6,8,10 \text {, and } 18 \text {; C-reactive protein, tumor necrosis } \\
\text { factor alpha- } \alpha\end{array}$ & $\checkmark$ & - & $\checkmark$ \\
\hline \multicolumn{5}{|l|}{ Outcome measures } \\
\hline Pain and symptoms & $\begin{array}{l}\text { McGill Pain Questionnaire, Seattle Angina Questionnaire-7, American } \\
\text { Heart Association Angina Log }\end{array}$ & $\checkmark$ & $\checkmark$ & $\checkmark$ \\
\hline Functional status and $\mathrm{HRQoL}^{\mathrm{c}}$ & $\begin{array}{l}\text { Seattle Angina Questionnaire-7, Physical Functioning and HRQoL sub- } \\
\text { scales }\end{array}$ & $\checkmark$ & $\checkmark$ & $\checkmark$ \\
\hline
\end{tabular}

${ }^{\mathrm{a}}$ Data were collected.

${ }^{\mathrm{b}}$ Data were not collected.

${ }^{\mathrm{c}}$ HRQoL: health-related quality of life.

\section{Randomization and Concealment}

The research specialist will screen and consent eligible individuals (Textbox 1). Potential participants will be informed that they will be randomized to either the acupuncture group (10-acupuncture session protocol, 2 treatments per week for 5 weeks) or the attention control group (2-hour video session once a week for 5 weeks). After informed consent is obtained, participants will be randomized to the acupuncture or attention control group using the randomization module on REDCap [41] based on the stratified, permuted block schedule prepared by the biostatistician. We are concealing the order of treatment arm assignment from the research associate responsible for recruiting and randomizing through the use of REDCap's restricted user rights. Our block sizes are concealed and our stratified approach also help to prevent research staff from guessing the sequence.

\section{Blinding}

For this feasibility study, because of the unique nature of acupuncture and the difficulty in blinding participant and acupuncturist (see Study Design), acupuncturists and participants will not be blinded to the group assignment. Although it would be optimal to have the research associate who collects outcome data blinded, this is not possible for the current feasibility protocol. Instead, we are using all self-administered outcome measures collected on a tablet computer. The co-principal investigators (co-PIs) and the biostatistician will remain blinded to group membership for data analyses.

\section{Research Protocol}

The research specialist will explain the nature of the study, risks, benefits, the voluntary nature of participation, and the right to discontinue participation at any time without consequences. After informed consent is obtained, participants will be randomized to the acupuncture or attention control group. All participants complete 4 measures at baseline: the Demographic Questionnaire, the MPQ [42], the Upper Body Outline [33], and the SAQ-7 [34]. Members of the acupuncture and control groups repeat the measures at the beginning of each session, either all 10 acupuncture sessions or following the 5 video sessions. All participants complete the AHA Angina Log (diary 
of symptoms) throughout the study, whenever they have angina symptoms. We will make copies of their paper log each time they come to the clinic in case they misplace their logs. Venipuncture will be performed at baseline and study completion.

\section{Acupuncture Intervention Protocol}

The acupuncturist will swab each point with alcohol. Needles will be inserted using a standardized TCM point prescription and retained for $30 \mathrm{~min}$. Each needle will be rotated 3 times to stimulate the $q i$ in the meridian; $10 \mathrm{~min}$ after insertion, $20 \mathrm{~min}$ after insertion, and just before removal at $30 \mathrm{~min}$. Needles will be inserted using the standards of clean needle technique established by the Council of Colleges of Acupuncture and
Oriental Medicine [43]. One size acupuncture needle, 0.25 diameter $\times 40 \mathrm{~mm}$ length, will be used for all participants receiving acupuncture. All acupuncture needles are sterile, disposable, and made of surgical stainless steel with stainless steel wound heads. Sessions will be repeated twice weekly (with at least 2 off days in between) for 5 weeks (10 sessions).

\section{Acupuncture Point Prescription for Angina}

The standardized point prescription (Figure 2) uses acupuncture points on the front of the body to enable participants, many of whom are acupuncture-naïve, to remain supine. This is aimed at reducing anxiety by enabling the participant to anticipate needle insertions.

Figure 2. Acupuncture point prescription for angina. Used with permission from the Journal of Chinese Medicine Publications [17].

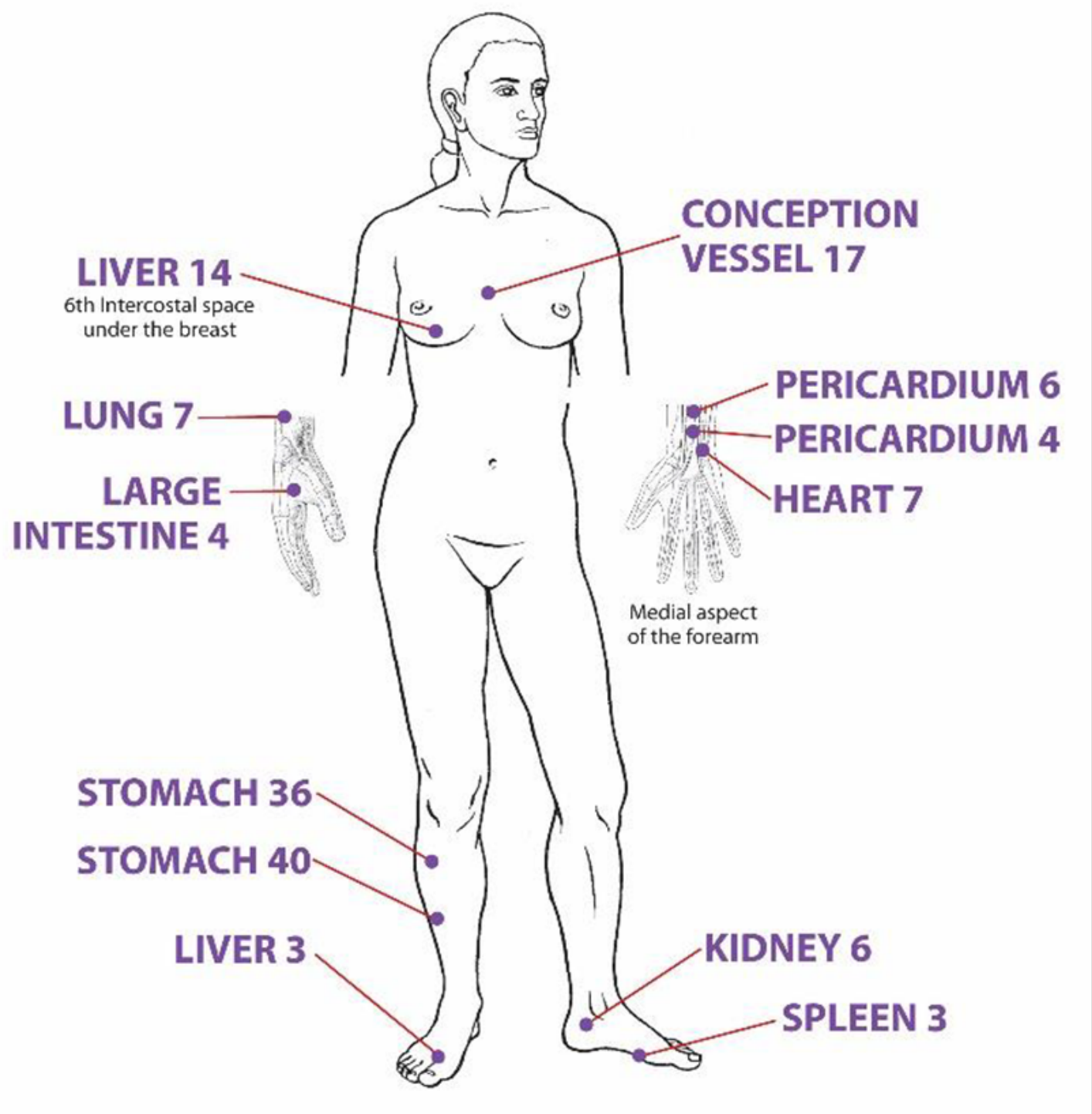




\section{Attention Control Health Videos Protocol}

The attention control group will watch health videos from the NOVA Science NOW series on Public Broadcasting System television. Topics do not contain content that could potentially improve pain. Videos are viewed from weeks 1 to 5 (5 time points) and equate to the time the experimental group receives acupuncture (10 hours) [44]. Titles include: "Can we live forever?," Cracking your genetic code," "Vaccines: Calling the shots," "How does the brain work?," "What are dreams?," "How smart can we get?," "Memory hackers, Cracking the code of life," and "Can Alzheimer's be stopped?." The videos are shown in our private clinical research rooms. Standardized content assures complete fidelity to the control protocol.

\section{Retention Strategies}

We anticipate an attrition rate of no more than $25 \%$. These numbers are based on accruals and withdrawals in our previous studies and our detailed retention plan. Participants will be engaged as active partners in the research by addressing possible benefits of acupuncture (experimental group) and non-pain-related health education (control group), as well as the importance of their contribution to science. To minimize participant attrition and avoid missing data, we are (1) offering the control group the acupuncture protocol, free of charge, at the conclusion of the study; (2) scheduling data collection at convenient times for participants, including weekend and evening appointments; (3) contacting patients using their preferred mode of communication (phone, text, and email) before each appointment; (4) providing patients with study team contact information; and (5) providing patient honoraria (US $\$ 10$ at each of 10 contact points for a total up to US \$100).

\section{Data Safety Monitoring}

The following data will be examined annually to evaluate safety issues every 6 months during the study: adverse events, attrition rate, reasons for attrition, and rate of adherence during the intervention. All adverse events will be monitored quarterly, but special attention will be given to serious adverse events that result in or require medical or surgical intervention to prevent death, a life-threatening situation, hospitalization, and a persistent or significant disability or incapacity. A Data and Safety Monitoring Committee (DMSC) has been appointed to provide oversight and monitoring of our data on an annual basis by individuals not directly associated with the study. The DMSC will consist of 2 scientists with expertise in RCT designs and a physician with expertise in the sample population. The co-PIs will meet with the DMSC annually and will present a written report as well as a verbal report. A report of the meeting will be submitted to the IRB. Additional meetings of the DMSC will be scheduled if the data are concerning or adverse events warrant more frequent review. If temporary or permanent suspension of the study occurs, the sponsoring institution IRBs and the National Institutes of Health will be notified.

\section{Adverse Events}

No serious adverse events are anticipated in this low risk study; however, all adverse events (eg, hospitalization or injury) and/or unanticipated problems (eg, exacerbation of angina or other acute illnesses) will be reported in writing to the IRB at UIC within 5 days of discovery of the incident. Serious or unexpected adverse events will be verbally reported within 24 to 48 hours.

\section{Quality Assurance}

Regular team meetings will be held for the duration of the research project, every 1 to 2 weeks. One principal investigator will chair these meetings. At the first several meetings, all recruitment and data collection procedures were reviewed with the research associate collecting data, and this process was and will be documented. To minimize potential for bias and to prevent contamination of the groups, 1 staff member will administer all instruments following acupuncture, and another staff member will administer all instruments following viewing of videos (for controls).

\section{Sample Size Calculation}

We are powered to detect recruitment, retention, completion, and acceptability rates for a future efficacy study, with a $95 \%$ 2-sided CI. With our planned sample size of 69 patients, the margin of error will be $9.5 \%$ if feasibility rates are in the desired range of $80 \%$ or greater; however, will be a maximum of $11.8 \%$ if these rates are as low as 50\% [45-47]. Similarly, we will compute a 2 -sided $95 \%$ CI for our effect size estimates, with a half-width of 0.557 standard deviation for posttest differences between groups assuming completion by $75 \%$ of participants [48]. With a sample size of 52 , we have $80 \%$ power to detect a large difference in mean change ( 0.7 standard deviation or greater) as statistically significant, assuming a repeated-measure correlation of 0.6 and a 2 -sided $t$ test with 0.05 significance level. We identified 1 similar study reporting continuous outcomes that found a large effect size of 0.85 for a pain reduction outcome and a 0.51 effect size for reduction in number of angina attacks per week [49].

\section{Statistical Analyses}

Our biostatistician will supervise data management and data analysis procedures. All data will be exported from REDCap and imported into SAS version 9.4 for cleaning and analysis. Descriptive statistics (frequencies, means, and standard deviations) and bivariate statistics by treatment group will be used to describe the sample and assess baseline differences. Nonnormal distributions may be optimally transformed using the Box-Cox method and/or analyzed using a comparable nonparametric test. Specific plans for analysis are as follows:

- Aim 1: To determine the feasibility (recruitment, completion, and acceptability rates of $80 \%$; retention rate of $75 \%$ ) of an RCT of acupuncture for stable angina. We will calculate the proportion of participants who are recruited, retained in the study, and complete the protocol. We will also calculate a $95 \%$ CI for these estimates. Acceptability will be examined by proportion of participants ( $\geq 80 \%$ ) scoring $\geq 16$ on the acceptability instrument. These statistics will be used to determine if protocol changes are needed to conduct a larger multisite trial.

- Aim 2: To estimate effect sizes for change from pre- to postintervention scores for pain/symptoms, inflammatory cytokines, functional status, and HRQoL between acupuncture and control groups. Effect size estimates from the literature were not of high quality or sufficiently 
comparable with power for an efficacy trial; thus, we are estimating effect sizes. However, if effects are large, our well-designed study may provide preliminary efficacy for the acupuncture intervention. We will analyze the trial's primary outcomes with a rigorous mixed-effect model for repeated measures recommended for primary analysis of clinical trials with continuous endpoints [50]. Biological sex will be included as a covariate. We will use intention-to-treat analyses, retaining all participants randomized to groups. Missing data will be addressed by using the full information maximum likelihood approach, which produces unbiased parameter estimates and SEs when data are missing at random [51,52]. Effect sizes and 95\% Cis will be calculated for pain, and symptoms will be reported via survey and diary, functional status, and HRQoL. We will use the model parameter estimate for treatment group-by-time (baseline to end point), divided by the control group's baseline standard deviation for a Glass delta effect size estimate using a bootstrap method for obtaining 95\% CIs [53].

- Exploratory aim: Explore between-group differences in levels of inflammatory biomarkers (IL-1 beta, IL- 2, IL-6, $I L-8, I L-10$, and IL-18; CRP; TNF alpha) from baseline to study completion ( 5 weeks). We will calculate effect size estimates and CIs using mixed models for repeated measures models as stated in Aim 2. We will also explore how changes in biomarkers relate to change in self-reported pain. These exploratory analyses will inform mechanism hypotheses in a future trial.

\section{Ethical Considerations}

The proposed protocol has only minimal potential physical or psychological risks to participants. The risks of this study are primarily those from acupuncture needle insertions including: soreness, minor bleeding, bruising after acupuncture needle removal, and fatigue after an acupuncture session. On the basis of findings from previous studies, the likelihood of these risks is very small [54]. There is also minor risk associated with venipuncture (for biomarkers). Collection of blood samples carry a small risk of pain or infection. Collection of data from questionnaires poses minimal time and emotional burden on participants. Finally, there may be some stress associated with keeping appointments for either the acupuncture intervention or the attention control video viewing session; this stress can be mitigated by emphasizing the voluntary nature of the visits, the expertise of the acupuncturist, and the comfortable and private surroundings.

Participants can refuse to participate in any or all of the study procedures or to withdraw from the study at any time. There are 3 categories of potential risk associated with this study: (1) Stress or emotional burden. Any psychological risks are likely to be related to items on the questionnaires that focus attention on symptoms and psychosocial variables. Participants may become uncomfortable thinking about their illness, treatment, or recovery. The major risk for this part of the study is the maintenance of confidentiality. Response burden for the participants is relatively low and will consist of time spent completing instruments, undergoing acupuncture, or watching health-related videos. If participants are tired or anxious, the sessions can be rescheduled for a later time; (2) Pain, discomfort, or physical harm. The risks from acupuncture needle insertions include soreness, minor bleeding, bruising after acupuncture needle removal, and fatigue after acupuncture. Blood will be obtained by venipuncture. Blood draws can cause syncope, temporary discomfort from the needle stick, bruising, and rarely infection. Patients will be encouraged to rest if necessary, and water and energy bars will be available for patients during each visit to the clinical research lab (for both groups); and (3) Loss of confidentiality. Every effort will be made to maintain patient privacy during data collection. In addition, a number of strategies to protect the privacy and confidentiality of each participant are in place including: proper and current Health Insurance Portability and Accountability Act (HIPAA) training and certification of all staff, compliance with HIPAA guidelines, storage of study data on a secure REDCap server and/or on a limited-access research drive, and placing angina logs and signed consent forms in a locked cabinet in the PIs office. All patients will participate only after written informed consent is obtained. The participants will retain a copy of the signed consent form. Guidelines for consent will be strictly followed according to the approval criteria of the IRB. All study data will be de-identified and stored in a private network drive at UIC that are accessible only to the co-PIs, biostatistician, and project director. Data will be collected on tablet computers, used solely for the study, and will be stored in a locked research office when not in use. Database access is limited to the co-PIs and the project director.

\section{Results}

This study has been funded over 2 years by the National Institutes of Health, National Institute for Nursing Research. We are currently recruiting and expect to have initial results by December 2020.

\section{Discussion}

\section{Overview}

The incidence of stable angina is projected to rise up to $18 \%$, with concomitant increases in comorbid conditions [55]. It is well known that angina patients complain of side effects from antianginal drugs, including nitroglycerin-related headaches, isosorbide-related dizziness and nausea/vomiting, and beta-blocker-related fatigue, nightmares, and depression which may lead to nonadherence and increased risk for future cardiac events $[5,56]$ A reduction in pain and associated symptoms of stable angina has the potential to improve functional status and HRQoL [3]. After beginning efficacy of this acupuncture protocol is established, future studies will include determining acupuncture from placebo effect, duration of effect, and optimal dosage effect [24,57]. These findings will then provide insight into the need for maintenance acupuncture treatments to reduce the chronic pain of stable angina.

\section{Conclusions}

This protocol represents the first step in examining acupuncture as a nonpharmacologic approach to the treatment of symptoms associated with stable angina. Acupuncture has been shown to 
have no major side effects [54,57]. Demonstrating the beginning efficacy of acupuncture for the reduction of symptoms of stable angina may shift its treatment from a purely Western model to a complementary model of care.

\section{Acknowledgments}

This study was made possible by Grant Number R21 NR017705-01 from the National Institutes of Health, National Institute of Nursing Research (NINR). Its contents are solely the responsibility of the authors and do not necessarily represent the official views of the NINR. The final peer-reviewed manuscript is subject to the National Institutes of Health Public Access Policy.

\section{Conflicts of Interest}

None declared.

\section{Multimedia Appendix 1}

Peer-reviewer report from the National Institutes of Health, National Institute of Nursing Research.

[PDF File (Adobe PDF File), 162KB-Multimedia Appendix 1]

\section{References}

1. Fox K, Garcia MA, Ardissino D, Buszman P, Camici PG, Crea F, Task Force on the Management of Stable Angina Pectoris of the European Society of Cardiology, ESC Committee for Practice Guidelines (CPG). Guidelines on the management of stable angina pectoris: executive summary: the task force on the management of stable angina pectoris of the European society of cardiology. Eur Heart J 2006 Jun;27(11):1341-1381. [doi: 10.1093/eurheartj/ehl001] [Medline: 16735367]

2. Benjamin EJ, Muntner P, Alonso A, Bittencourt MS, Callaway CW, Carson AP, American Heart Association Council on Epidemiology and Prevention Statistics Committee and Stroke Statistics Subcommittee. Heart disease and stroke statistics-2019 update: a report from the American Heart Association. Circulation 2019 Dec 5;139(10):e56-528. [doi: 10.1161/CIR.0000000000000659] [Medline: 30700139]

3. Camm AJ, Manolis A, Ambrosio G, Daly C, Komajda M, Lopez de Sa E, et al. Unresolved issues in the management of chronic stable angina. Int J Cardiol 2015 Dec 15;201:200-207. [doi: 10.1016/j.ijcard.2015.08.045] [Medline: 26298381]

4. Fraker Jr TD, Fihn SD, 2002 Chronic Stable Angina Writing Committee, American College of Cardiology, American Heart Association, Gibbons RJ, et al. 2007 chronic angina focused update of the ACC/AHA 2002 guidelines for the management of patients with chronic stable angina: a report of the American College of Cardiology/American Heart Association Task Force on Practice Guidelines Writing Group to develop the focused update of the 2002 guidelines for the management of patients with chronic stable angina. J Am Coll Cardiol 2007 Dec 4;50(23):2264-2274 [FREE Full text] [doi: 10.1016/j.jacc.2007.08.002] [Medline: 18061078 ]

5. Xu L, Xu H, Gao W, Wang W, Zhang H, Lu DP. Treating angina pectoris by acupuncture therapy. Acupunct Electrother Res 2013;38(1-2):17-35. [doi: 10.3727/036012913X13831831849457] [Medline: 23724696]

6. Fang J, Jin Z, Wang Y, Li K, Kong J, Nixon EE, et al. The salient characteristics of the central effects of acupuncture needling: limbic-paralimbic-neocortical network modulation. Hum Brain Mapp 2009 Apr;30(4):1196-1206. [doi: $\underline{10.1002 / \mathrm{hbm} .20583}$ ] [Medline: $\underline{18571795]}$

7. Harris RE, Zubieta JK, Scott DJ, Napadow V, Gracely RH, Clauw DJ. Traditional Chinese acupuncture and placebo (sham) acupuncture are differentiated by their effects on mu-opioid receptors (MORs). Neuroimage 2009 Sep;47(3):1077-1085 [FREE Full text] [doi: 10.1016/j.neuroimage.2009.05.083] [Medline: 19501658]

8. Headrick JP, Pepe S, Peart JN. Non-analgesic effects of opioids: cardiovascular effects of opioids and their receptor systems. Curr Pharm Des 2012;18(37):6090-6100. [doi: 10.2174/138161212803582360] [Medline: 22747541]

9. Saraiva J, Oliveira SM, Rocha-Sousa A, Leite-Moreira A. Opioid receptors and preconditioning of the heart. Rev Port Cardiol 2004 Oct;23(10):1317-1333. [Medline: 15641296 ]

10. Holaday JW. Cardiovascular effects of endogenous opiate systems. Annu Rev Pharmacol Toxicol 1983;23:541-594. [doi: 10.1146/annurev.pa.23.040183.002545] [Medline: 6307129]

11. Chae Y, Chang DS, Lee SH, Jung W, Lee I, Jackson S, et al. Inserting needles into the body: a meta-analysis of brain activity associated with acupuncture needle stimulation. J Pain 2013 Mar;14(3):215-222. [doi: 10.1016/j.jpain.2012.11.011] [Medline: 23395475]

12. Hui KK, Marina O, Liu J, Rosen BR, Kwong KK. Acupuncture, the limbic system, and the anticorrelated networks of the brain. Auton Neurosci 2010 Oct 28;157(1-2):81-90 [FREE Full text] [doi: 10.1016/j.autneu.2010.03.022] [Medline: 20494627]

13. Smith Jr FW. Acupuncture for cardiovascular disorders. Probl Vet Med 1992 Mar;4(1):125-131. [Medline: 1581649$]$

14. Ren X, Guo M, Zhao Y, Ding X, Li C, Ji B, et al. [Effects of electroacupuncture on pain reactions, expression of spinal kappa-opioid receptor and contents of enkephalin and beta-endorphin in periaqueductal gray of midbrain in dysmenorrhea model rats]. Zhen Ci Yan Jiu 2012 Feb;37(1):1-7. [Medline: 22574561] 
15. Chavan SS, Tracey KJ. Regulating innate immunity with dopamine and electroacupuncture. Nat Med 2014 Mar;20(3):239-241. [doi: 10.1038/nm.3501] [Medline: 24603793]

16. Kaptchuk TJ. The Web That Has No Weaver: Understanding Chinese Medicine. Chicago: Congdon \& Weed; 1983.

17. Deadman P, Al-Khafaji M, Baker K. A Manual Of Acupuncture. Second Edition. Vista, CA: Journal of Chinese Medicine Publications; 1998.

18. Unschuld PU, Tessenow H. Huang Di Nei Jing Su Wen: An Annotated Translation Of Huang Di's Inner Classic - Basic Questions: 2 Volumes. Berkeley, CA: University Of California Press; 2011.

19. Zhong Y, Xue JC. In: Wiseman N, editor. Fundamentals of Chinese Medicine. Brookline, MA: Paradigm Press; 1995.

20. Guang-Ren S, Eisenstarck D, Zhang QR. Fundamentals of Chinese Medicine. First Edition. China: People's Medical Pubblishing House; 2014.

21. Yu C, Ji K, Cao H, Wang Y, Jin HH, Zhang Z, et al. Effectiveness of acupuncture for angina pectoris: a systematic review of randomized controlled trials. BMC Complement Altern Med 2015 Mar 28;15:90 [FREE Full text] [doi: 10.1186/s12906-015-0586-7] [Medline: 25888363]

22. Zhang Z, Chen M, Zhang L, Zhang Z, Wu W, Liu J, et al. Meta-analysis of acupuncture therapy for the treatment of stable angina pectoris. Int J Clin Exp Med 2015;8(4):5112-5120 [FREE Full text] [Medline: 26131084]

23. Takakura N, Yajima H. A double-blind placebo needle for acupuncture research. BMC Complement Altern Med 2007 Oct 10;7:31 [FREE Full text] [doi: 10.1186/1472-6882-7-31] [Medline: 17925042]

24. Schlaeger JM, Takakura N, Yajima H, Takayama M, Steffen AD, Gabzdyl EM, et al. Double-blind acupuncture needles: a multi-needle, multi-session randomized feasibility study. Pilot Feasibility Stud 2018;4:72 [FREE Full text] [doi: 10.1186/s40814-018-0265-9] [Medline: 29686883]

25. Zhang SJ. [Origin and development of Ashi point locating method]. Zhongguo Zhen Jiu 2013 Feb;33(2):165-167. [Medline: 23620951]

26. Zhu D, Gao Y, Chang J, Kong J. Placebo acupuncture devices: considerations for acupuncture research. Evid Based Complement Alternat Med 2013;2013:628907 [FREE Full text] [doi: 10.1155/2013/628907] [Medline: 23840261]

27. Campeau L. Letter: grading of angina pectoris. Circulation 1976 Sep;54(3):522-523. [doi: 10.1161/circ.54.3.947585Circulation] [Medline: 947585$]$

28. Melzack R. The McGill pain questionnaire: major properties and scoring methods. Pain 1975 Sep;1(3):277-299. [doi: 10.1016/0304-3959(75)90044-5] [Medline: 1235985]

29. Bruce J, Drury N, Poobalan AS, Jeffrey RR, Smith WC, Chambers WA. The prevalence of chronic chest and leg pain following cardiac surgery: a historical cohort study. Pain 2003 Jul;104(1-2):265-273. [doi: 10.1016/S0304-3959(03)00017-4] [Medline: 12855337]

30. Watt-Watson J, Stevens B, Garfinkel P, Streiner D, Gallop R. Relationship between nurses' pain knowledge and pain management outcomes for their postoperative cardiac patients. J Adv Nurs 2001 Nov;36(4):535-545. [Medline: 11703548]

31. Kimble LP, McGuire DB, Dunbar SB, Fazio S, De A, Weintraub WS, et al. Gender differences in pain characteristics of chronic stable angina and perceived physical limitation in patients with coronary artery disease. Pain 2003 Jan;101(1-2):45-53 [FREE Full text] [doi: 10.1016/S0304-3959(02)00319-6] [Medline: 12507699]

32. Eisenberg E, Pultorak Y, Pud D, Bar-El Y. Prevalence and characteristics of post coronary artery bypass graft surgery pain (PCP). Pain 2001 May;92(1-2):11-17. [doi: 10.1016/S0304-3959(00)00466-8] [Medline: 11323122]

33. Everts B, Karlson BW, Währborg P, Hedner T, Herlitz J. Localization of pain in suspected acute myocardial infarction in relation to final diagnosis, age and sex, and site and type of infarction. Heart Lung 1996;25(6):430-437. [doi: 10.1016/S0147-9563(96)80043-4] [Medline: 8950121]

34. Chan PS, Jones PG, Arnold SA, Spertus JA. Development and validation of a short version of the Seattle angina questionnaire. Circ Cardiovasc Qual Outcomes 2014 Sep;7(5):640-647 [FREE Full text] [doi: 10.1161/CIRCOUTCOMES.114.000967] [Medline: 25185249]

35. Spertus JA, Winder JA, Dewhurst TA, Deyo RA, Prodzinski J, McDonell M, et al. Development and evaluation of the Seattle angina questionnaire: a new functional status measure for coronary artery disease. J Am Coll Cardiol 1995 Feb;25(2):333-341 [FREE Full text] [doi: 10.1016/0735-1097(94)00397-9] [Medline: 7829785]

36. Arnold SV, Kosiborod M, Li Y, Jones PG, Yue P, Belardinelli L, et al. Comparison of the Seattle angina questionnaire with daily angina diary in the TERISA clinical trial. Circ Cardiovasc Qual Outcomes 2014 Nov;7(6):844-850. [doi: 10.1161/CIRCOUTCOMES.113.000752] [Medline: 25249560]

37. Yiu EM, Chan KM, Li NY, Tsang R, Verdolini AK, Kwong E, et al. Wound-healing effect of acupuncture for treating phonotraumatic vocal pathologies: a cytokine study. Laryngoscope 2016 Jan;126(1):E18-E22 [FREE Full text] [doi: 10.1002/lary.25483] [Medline: 26227080]

38. Nager AL, Kobylecka M, Pham PK, Johnson L, Gold JI. Effects of acupuncture on pain and inflammation in pediatric emergency department patients with acute appendicitis: a pilot study. J Altern Complement Med 2015 May;21(5):269-272. [doi: 10.1089/act.2015.29027.sho] [Medline: 25875844]

39. Yeh CH, Chien LC, Albers KM, Ren D, Huang LC, Cheng B, et al. Function of auricular point acupressure in inducing changes in inflammatory cytokines during chronic low-back pain: a pilot study. Medical Acupuncture 2014 Feb;26(1):31-39. [doi: $\underline{10.1089 / \text { acu.2013.1015] }}$ 
40. Omland T, White HD. State of the art: blood biomarkers for risk stratification in patients with stable ischemic heart disease. Clin Chem 2017 Jan;63(1):165-176 [FREE Full text] [doi: 10.1373/clinchem.2016.255190] [Medline: 27815307]

41. Harris PA, Taylor R, Thielke R, Payne J, Gonzalez N, Conde JG. Research electronic data capture (REDCap)--a metadata-driven methodology and workflow process for providing translational research informatics support. J Biomed Inform 2009 Apr;42(2):377-381 [FREE Full text] [doi: 10.1016/j.jbi.2008.08.010] [Medline: 18929686 ]

42. Melzack R. The short-form McGill pain questionnaire. Pain 1987 Aug;30(2):191-197. [doi: 10.1016/0304-3959(87)91074-8] [Medline: 3670870]

43. Brazil J, Mitchell B, Tu A, Skelton W. Clean Needle Technique Manual for Acupuncturists: Guidelines and Standards for the Clean and Safe Clinical Practice of Acupuncture. Washington, DC: National Acupuncture Foundation; 1997.

44. Smith MT, Finan PH, Buenaver LF, Robinson M, Haque U, Quain A, et al. Cognitive-behavioral therapy for insomnia in knee osteoarthritis: a randomized, double-blind, active placebo-controlled clinical trial. Arthritis Rheumatol 2015 May;67(5):1221-1233 [FREE Full text] [doi: 10.1002/art.39048] [Medline: 25623343]

45. Arnold DM, Burns KE, Adhikari NK, Kho ME, Meade MO, Cook DJ, McMaster Critical Care Interest Group. The design and interpretation of pilot trials in clinical research in critical care. Crit Care Med 2009 Jan;37(1 Suppl):S69-S74. [doi: 10.1097/CCM.0b013e3181920e33] [Medline: 19104228]

46. Fleiss JL, Levin B, Paik MC. Statistical Methods for Rates \& Proportions. Hoboken, New Jersey: John Wiley \& Sons; 2013.

47. Newcombe RG. Two-sided confidence intervals for the single proportion: comparison of seven methods. Stat Med 1998 Apr 30;17(8):857-872. [doi: 10.1002/(SICI)1097-0258(19980430)17:8<857::AID-SIM777>3.0] [Medline: 9595616]

48. Zar JH. Biostatistical Analysis. Second Edition. Englewood, Colorado: Prentice Hall; 1984.

49. Richter A, Herlitz J, Hjalmarson A. Effect of acupuncture in patients with angina pectoris. Eur Heart J 1991 Feb;12(2):175-178. [doi: 10.1093/oxfordjournals.eurheartj.a059865] [Medline: 2044550]

50. Mallinckrod CH, Lane PW, Schnell D, Peng Y, Mancuso JP. Recommendations for the primary analysis of continuous endpoints in longitudinal clinical trials. Drug Inf J 2008 Jul;42(4):303-319 [FREE Full text] [doi: 10.1177/009286150804200402]

51. Graham JW. Missing data analysis: making it work in the real world. Annu Rev Psychol 2009;60:549-576. [doi: 10.1146/annurev.psych.58.110405.085530] [Medline: 18652544 ]

52. Press SJ. Subjective and Objective Bayesian Statistics: Principles, Models, and Applications. Hoboken, New Jersey: John Wiley \& Sons; 2009.

53. Friedmann PD, Rose JS, Swift R, Stout RL, Millman RP, Stein MD. Trazodone for sleep disturbance after alcohol detoxification: a double-blind, placebo-controlled trial. Alcohol Clin Exp Res 2008 Sep;32(9):1652-1660 [FREE Full text] [doi: 10.1111/j.1530-0277.2008.00742.x] [Medline: 18616688]

54. MacPherson H, Thomas K, Walters S, Fitter M. The York acupuncture safety study: prospective survey of 34000 treatments by traditional acupuncturists. Br Med J 2001 Sep 1;323(7311):486-487 [FREE Full text] [doi: 10.1136/bmj.323.7311.486] [Medline: 11532841]

55. Go AS, Mozaffarian D, Roger VL, Benjamin EJ, Berry JD, Blaha MJ, American Heart Association Statistics Committee and Stroke Statistics Subcommittee. Heart disease and stroke statistics--2014 update: a report from the American Heart Association. Circulation 2014 Jan 21;129(3):e28-292 [FREE Full text] [doi: 10.1161/01.cir.0000441139.02102.80] [Medline: 24352519]

56. Fihn SD, Blankenship JC, Alexander KP, Bittl JA, Byrne JG, Fletcher BJ, American College of Cardiology/Americal Heart Association Task Force on Practice Guidelines, American Association for Thoracic Surgery, Preventive Cardiovascular Nurses Association, Society for Cardiovascular Angiography and Interventions, Society of Thoracic Surgeons. 2014 ACC/AHA/AATS/PCNA/SCAI/STS focused update of the guideline for the diagnosis and management of patients with stable ischemic heart disease: a report of the American College of Cardiology/American Heart Association Task Force on Practice Guidelines, and the American Association for Thoracic Surgery, Preventive Cardiovascular Nurses Association, Society for Cardiovascular Angiography and Interventions, and Society of Thoracic Surgeons. J Thorac Cardiovasc Surg 2015 Mar;149(3):e5-23 [FREE Full text] [doi: 10.1016/j.jtcvs.2014.11.002] [Medline: 25827388]

57. Vincent C. The safety of acupuncture. Br Med J 2001 Sep 1;323(7311):467-468 [FREE Full text] [doi: 10.1136/bmj.323.7311.467] [Medline: 11532826 ]

\section{Abbreviations}

AHA: American Heart Association

CCS: Canadian Cardiovascular Society

Co-PI: co-principal investigator

CRP: C-reactive protein

DMSC: Data and Safety Monitoring Committee

HIPAA: Health Insurance Portability and Accountability Act

HRQoL: health-related quality of life 
IL: interleukin

IRB: Institutional Review Board

MPQ: McGill Pain Questionnaire

RCT: randomized controlled trial

SAQ-7: Seattle Angina Questionnaire-7

TCM: traditional Chinese medicine

TNF: tumor necrosis factor

UIC: University of Illinois at Chicago

\author{
Edited by G Eysenbach; submitted 13.05.19; peer-reviewed by A Eckhardt, L Davis, I Quah-Smith; comments to author 15.06.19; \\ accepted 27.06.19; published 29.07.19 \\ Please cite as: \\ Schlaeger J, Cai HY, Steffen AD, Angulo V, Shroff AR, Briller JE, Hoppensteadt D, Uwizeye G, Pauls HA, Takayama M, Yajima H, \\ Takakura N, DeVon HA \\ Acupuncture to Improve Symptoms for Stable Angina: Protocol for a Randomized Controlled Trial \\ JMIR Res Protoc 2019;8(7):e14705 \\ URL: http://www. researchprotocols.org/2019/7/e14705/ \\ doi: $10.2196 / 14705$ \\ PMID: $\underline{31359872}$
}

CJudith Schlaeger, Hui Yan Cai, Alana D Steffen, Veronica Angulo, Adhir R Shroff, Joan E Briller, Debra Hoppensteadt, Glorieuse Uwizeye, Heather A Pauls, Miho Takayama, Hiroyoshi Yajima, Nobuari Takakura, Holli A DeVon. Originally published in JMIR Research Protocols (http://www.researchprotocols.org), 29.07.2019. This is an open-access article distributed under the terms of the Creative Commons Attribution License (https://creativecommons.org/licenses/by/4.0/), which permits unrestricted use, distribution, and reproduction in any medium, provided the original work, first published in JMIR Research Protocols, is properly cited. The complete bibliographic information, a link to the original publication on http://www.researchprotocols.org, as well as this copyright and license information must be included. 